\title{
Atomic-resolution differential phase contrast STEM on ferroelectric materials: A mean-field approach
}

\author{
M. Campanini $\odot,{ }^{1,}{ }^{*}$ K. Eimre $\odot,{ }^{2}$ M. Bon $\odot,{ }^{1}$ C. A. Pignedoli, ${ }^{2}$ M. D. Rossell $\odot,{ }^{1}$ and R. Erni ${ }^{1}$ \\ ${ }^{1}$ Electron Microscopy Center, Swiss Federal Laboratories for Materials Science and Technology, Empa, Ueberlandstrasse 129, \\ 8600 Dübendorf, Switzerland \\ ${ }^{2}$ nanotech@surfaces Laboratory, Swiss Federal Laboratories for Materials Science and Technology, Empa, Ueberlandstrasse 129, \\ 8600 Dübendorf, Switzerland
}

(Received 26 March 2020; accepted 5 May 2020; published 22 May 2020)

\begin{abstract}
The ultimate challenge in the investigation of ferroelectric properties lies in the quantitative measurements of their polarization at the unit cell scale. Such investigations are commonly performed using an indirect approach, by measuring the atomic displacements from atomic resolution images. Differential phase-contrast (DPC) scanning transmission electron microscopy (STEM) allows mapping the electric field with atomic resolution. This unique capability offers a direct way to study the polar properties in ferroelectrics. However, the effects of ferroelectric polarization on the contrast of high-resolution DPC-STEM imaging have not been addressed so far. In this work, we perform a theoretical study on the origin of the DPC-STEM contrast in ferroelectric materials and propose a modified multislice algorithm for STEM image simulations. Our results demonstrate that the mesoscopic polarization induces asymmetries in the detected electric fields, which are in line with our previous experimental observations. Moreover, we discuss the dependence of the DPC-STEM sensitivity on the polar field amplitude, specimen thickness, and defocus, and provide a route to discriminate between mesoscopic polarization and specimen misorientation.
\end{abstract}

DOI: 10.1103/PhysRevB.101.184116

\section{INTRODUCTION}

The advent of aberration correctors marked an unprecedented revolution in the resolving capability of modern scanning transmission electron microscopy (STEM), allowing imaging techniques at atomic and, more recently, at subangstrom resolution [1,2]. In particular, in STEM-based techniques, a focused probe with a lateral size typically smaller than $1 \AA$ is used to perform a raster scan of the specimen and the signal generated by the beam-specimen interaction is integrated on the conjugated plane employing annular detectors extending over different angular ranges. The most common STEM configuration consists of an array of detectors including, e.g., high-angle annular dark-field (HAADF), annular dark-field (ADF), and (annular) bright-field (BF) detectors [3].

Besides these conventional imaging techniques, which make use of integrating diffraction intensity over an annular area to generate a single-value signal for each probe position during the scan, new imaging techniques that are sensitive to the azimuthal variation of the convergent beam electron diffraction (CBED) pattern are gathering a rapidly growing interest. These new imaging techniques include differential phase-contrast (DPC) STEM [4] and more general fourdimensional (4D) STEM [5], which are realized by using either segmented annular detectors or pixel-array cameras to record the differential signal between pairs of opposite

*Corresponding author: marco.campanini@empa.ch detector segments or the entire CBED pattern, respectively. Although not entirely new (see, e.g., Rose [6], Dekkers and de Lang [7], Haider et al. [8], Hammel and Rose [9]), these detection strategies allow for exploring additional contrast mechanisms that hitherto have been buried by the azimuthal integration of conventional annular detectors. The key point for the success of such techniques relies on the correlation between the variation in the intensity distribution within the transmitted disk and relevant sample properties, such as local changes of the specimen composition [10,11], structure and strains [12-16], electric/magnetic fields in the specimen plane [17-28]. Remarkably, performing these techniques in STEM mode allows for combining the high spatial resolution typical of STEM with a large field of view, a condition not easily achievable by other phase-contrast techniques like, e.g., by electron holography.

One of the most striking applications of differential phasecontrast STEM is the detection of the electric fields within the specimen. Under a semi-classical approximation of the electron beam propagation, the effect of an electric field in the specimen plane is simply pictured as a deflection of the electron beam due to the electric component of the Lorentz force (rigid-shift model [4,29]). This simplified model has been successfully applied to interpret experimental DPCSTEM data at low/medium resolution, i.e., with a probe size spanning over a few unit cells. This condition assures that the electric field varies weakly over the lateral size of the probe [30]. Indeed, ferroelectric domains in $\mathrm{BaTiO}_{3}$ and $\mathrm{BiFeTi}_{3} \mathrm{O}_{15}$ were investigated by analyzing the contrast variation in the DPC-STEM signals $[4,28]$, showing the great potential of the 
DPC techniques for the investigation of ferroelectric oxides. Despite the success of this simple model in describing the large scale polar pattern in functional oxides, a full dynamical description of the electron propagation is necessary for the interpretation of the differential phase-contrast STEM signal at high-resolution where the condition of a weakly varying signal across the electron probe is not met. Indeed, in the case of atomic resolution experiments (i) the atomic electric field varies very rapidly over distances which are comparable to the lateral size of the electron probe and (ii) the dynamical effects in the electron diffraction processes are not negligible for typical thicknesses of real specimens. [26]

In a previous work, we observed a variation of the atomicresolution DPC-STEM signal in differently polarized regions of a complex ferroelectric structure naturally formed in a Cadoped $\mathrm{BiFeO}_{3}$ thin film. [31] This finding proved that highresolution DPC-STEM-although requiring a more complex description than the rigid-shift model-is sensitive to polar fields in ferroelectric materials and they appear as asymmetries of the electric field detected by DPC-STEM. However, a complete understanding of these first experimental observations has not yet been put forward, rather giving rise to various puzzling questions about the possible exploitation of this technique for the investigation of functional materials, like ferroelectrics and multiferroics. In particular, understanding the origin of the atomic-resolution DPC-STEM signal and its applicability to relevant materials-e.g., DPC-STEM sensitivity limits, the role of dynamical diffraction and specimen tilt- -still constitute open challenges.

Therefore we present here a comprehensive theoretical work based on modified multislice simulations [32] in which we take into account the contribution of the ferroelectric polarization in the calculation of the DPC-STEM signal. Firstly, we show a comparison of two different approaches to generate the crystal potential, i.e., the independent-atom model (IAM) and density functional theory (DFT) calculations and discuss how to include the contribution of the ferroelectric polarization in the multislice algorithm preserving the periodicity of the electrostatic crystal potential. Then, we compare the highresolution signals generated by the multislice simulations in the presence or absence of a mesoscopic polar field. Finally, the role of crystal tilt is discussed by simulating and comparing the effects induced by the sample misorientation and the mesoscopic polarization.

\section{MATERIAL AND METHODS}

\section{A. Bismuth ferrite $\left(\mathrm{BiFeO}_{3}\right)$}

In this work, we report a case study on the origin of the atomic-resolution DPC-STEM signal in a ferroelectric material, focusing on bismuth ferrite $\left(\mathrm{BiFeO}_{3}, \mathrm{BFO}\right)$. $\mathrm{BFO}$ has been widely studied for being one among the few materials exhibiting spontaneous multiferroicity at room temperature and represents an archetype ferroelectric material thanks to its robust ferroelectric polarization as high as $60-100 \mu \mathrm{C} \mathrm{cm}^{-2}$ in both bulk [33] and thin films [34-37].

BFO shows ferroelectricity and antiferromagnetic (G-type) ordering at room temperature, with Curie $\left(T_{\mathrm{C}}\right)$ and Neel $\left(T_{\mathrm{N}}\right)$ temperatures at 1100 and $653 \mathrm{~K}$, respectively. The bismuth ferrite's large polarization is generated by the localized $6 s^{2}$ lone pair on the $\mathrm{Bi}$ atoms [38], which repulses the $\mathrm{O}$ anions and induces an off-centering of the center of mass of positive and negative charges. Interestingly, the polar state of BFO can be precisely controlled by substitutional doping, e.g., by replacing the lone-pair active $\mathrm{Bi}^{3+}$ cations with different cations (like $\mathrm{Ca}^{2+}$ ) that do not show a localized electron lone pair. Indeed, our earlier studies on $\mathrm{Bi}_{0.8} \mathrm{Ca}_{0.2} \mathrm{FeO}_{3}$ showed that layering of the $\mathrm{Ca}$ doping promotes the formation of a complex polar pattern where strongly polar Ca-depleted regions alternate with areas where, due to the higher $\mathrm{Ca}$ doping, the polarization is quenched [31]. In the polar areas, a polarization as high as $80 \mu \mathrm{Ccm}^{-2}$ was estimated from the quantitative measurements of the atomic displacements obtained from the HAADF images.

The precise control of ferroelectric polarization achievable by the layered dopant profile enabled in turn to have a prototypal system for assessing the sensitivity of DPCSTEM to polar fields. Indeed, we observed that asymmetries in the atomic electric fields are visible in the polar regions corresponding to the $\mathrm{Ca}$ depleted areas in the complex layered ferroelectric structure [31]. However, due to the complexity of the electronic structure of BFO and the significant role of the dynamical effects on the electron beam propagation - that cannot be neglected for specimen thicknesses in the range of tens of nanometers-the quantitative interpretation of the experimental DPC data requires a comprehensive comparison with image simulations.

\section{B. Ferroelectric polarization: Modeling and implementation}

The modeling of a ferroelectric crystal potential-needed for the multislice simulations-requires that three main contributions are considered: (1) the potential of the atoms; (2) the potential due to the microscopic dipoles; and (3) the potential due to the mesoscopic field $\left(E_{\mathrm{P}}\right)$, which can be alternatively seen as the field generated by the bound charges. The potential due to the atoms constituting the crystal structure is periodic and can be computed either by the IAM or DFT approaches starting from the unit cell atomic coordinates. The details about the structural model and the atomic potential calculation for the IAM and DFT cases will be discussed in Secs. II D and II F, respectively.

In proper ferroelectrics, the material polarization arises as a consequence of the atomic displacements. In particular, an electric dipole is generated at the unit cell scale because the centers of the positive and negative charges are at a finite distance, which is usually in the picometer range [39]. Since such a local electric dipole is created due to the atomic displacements, its local effect is encoded in the coordinates of the atoms within the unit cell. Therefore the tiny potential contribution due to microscopic dipoles is intrinsically included in the IAM and DFT potentials.

A comprehensive description of the system, however, requires that we introduce the effects of the mesoscopic field due to the polarization, i.e., the contribution of the surrounding crystal whose aligned electric dipoles generate a polar field throughout the entire system. This additional contribution has been already pointed out in a previous work about the calculation of the phase shift due to a ferroelectric crystal in 
electron holography [40]. The implementation of the mesoscopic field is also particularly important in the simulation of high-resolution data, where only a very limited portion of the crystal is used in the calculations of the potential slices.

Within a ferroelectric domain, whose lateral size is commonly in a range that spans from tens to hundreds of nanometers [41], the mesoscopic polarization can be considered uniform over several nanometers. Therefore the polarization value can be assumed constant on the length scale of several atomic distances. Under this condition, we can conveniently describe the mesoscopic polar field by using a mean-field approach, i.e., we can represent its contribution as an additive constant field, whose value is related to the average material polarization, which sums up to the contributions due to the atomic potentials and the local microscopic dipoles [Fig. 1(a)].

As a first step, we need to find an advantageous way to implement this mean-field approach in the multislice image simulation routine. The multislice algorithm is based on the iterative calculation of the interaction of the beam with the electrostatic potential of a slice and the subsequent free-space Fresnel propagation until the next slice [32]. Therefore two different routes are possible, i.e., including the contribution of polarization (i) in the potential slices or (ii) in the free-space propagation.

In the first approach, we can introduce the mesoscopic polarization in the expression used for the calculation of the projected potential slices:

$$
V_{i}^{\prime}\left(\mathbf{r}, z_{i}\right)=\frac{1}{\Delta z} \int_{z_{i}}^{z_{i}+\Delta z}\left[V(\mathbf{r}, z)+V_{P}(\mathbf{r}, z)\right] d z
$$

where $V_{i}^{\prime}\left(\mathbf{r}, z_{i}\right)$ is the total projected electrostatic potential at the position $\mathbf{r}$ for the $i$ th slice, $V(\mathbf{r}, z)$ and $V_{P}(\mathbf{r}, z)$ are respectively the potential due to the crystal lattice and the mesoscopic ferroelectric polarization at the coordinate $(\mathbf{r}, z)$, $z$ is the coordinate along the beam propagation direction, $\Delta z$ is the thickness of the slice. The $V_{P}$ term can be expressed as the inverse gradient of the electric field due to the ferroelectric polarization:

$$
V_{P}(\mathbf{r}, z)=-\nabla_{r}^{-1} E_{P}(z)=-\int E_{P}(z) d \mathbf{r} .
$$

In ferroelectric materials, when no external field is applied, the microscopic electric field due to the remnant polarization $\left(P_{R}\right)$ is given by

$$
E_{P}(z)=-\frac{P_{R}(z)}{\varepsilon_{0}},
$$

where $\varepsilon_{0}=8.854 \times 10^{-12} \mathrm{CV}^{-1} \mathrm{~m}^{-1}$ is the vacuum permittivity. Using Eqs. (2) and (3) it is possible to calculate the potential due to the ferroelectric polarization for each point (r, $z$ ) within the specimen and then include this term in the calculation of the potential slices defined in Eq. (1). Performing the calculation, we promptly find that the mesoscopic polar field contribution to the electrostatic potential has a linear dependency on $\mathbf{r}$, which can be added to the potential slices used for the conventional multislice simulations [Fig. 1(b)]. Nevertheless, such an additive term is responsible for a disruption in the symmetry of the projected potential, whose effect
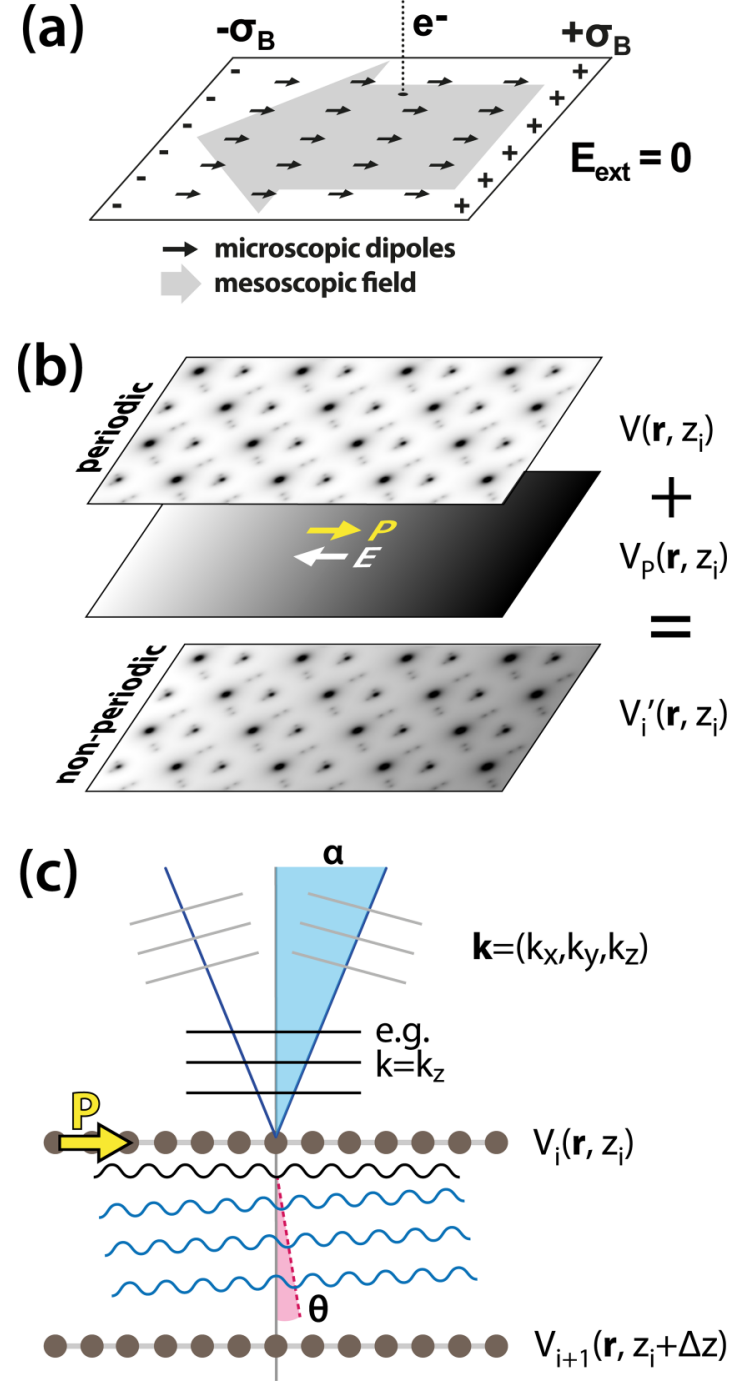

FIG. 1. (a) Sketch of a ferroelectric crystal slab when no external field is applied. The small black arrows give the microscopic dipoles of the $\mathrm{BiFeO}_{3}$ pseudocubic unit cells, while the large grey arrow depicts the mesoscopic field due to whole the crystal polarization. One possible electron trajectory is given by the black dashed line. (b) Effect of a uniform ferroelectric polarization on the potential slices for multislice simulations. An additive linear term induces disruption of the periodicity of the potential slices. (c) Modified multislice approach including the interaction of the electrons with a transverse electric field in the free-space propagation. After interacting with the potential slice $V_{i}$, the electron wavefunction is propagated in the free-space until the next potential slice $V_{i+1}$, applying a fractional beam tilt corresponding to the effect induced by the ferroelectric polarization of one slice.

is to produce nonperiodic boundaries that certainly affect the Fourier-transform-based multislice calculations.

As a second approach, we can alternatively describe the electron propagation through the polar crystal by introducing the mesoscopic polar field at a different step of the multislice simulation routine, i.e., the free-space propagation. Indeed, under a semiclassical approximation, the effect of an electric field on an electron can be expressed by the angular deviation induced in its trajectory due to the electric component of 

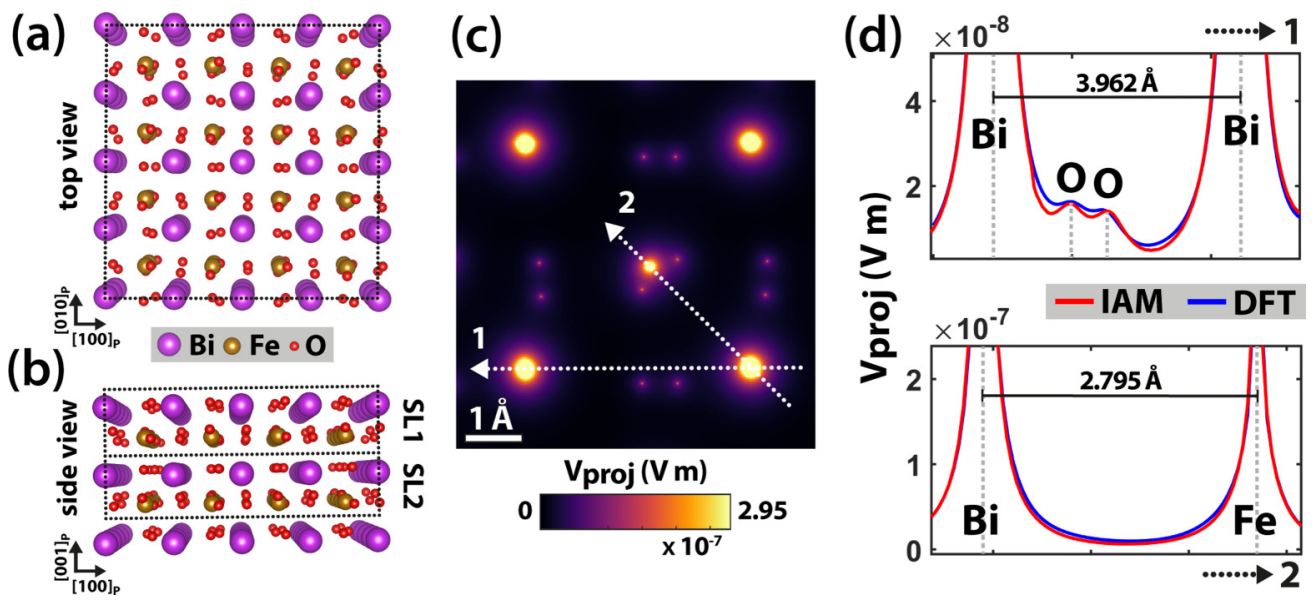

FIG. 2. Crystal structure of $\mathrm{BiFeO}_{3}$ viewed along (a) the $[001]_{P}$ (top view) and (b) the $[010]_{P}$ (side view) directions. The lateral size of the crystal is $15.874 \AA$. Along the $z$ direction, the crystal is 7.937- $\AA$ thick. Two slices (SL1, SL2) are cut for generating the projected potential. (c) Total projected electrostatic potential $\left(V_{\mathrm{SL} 1}+V_{\mathrm{SL} 2}\right)$ as obtained from the independent atom model. (d) Line profiles of the projected electrostatic potential - as obtained from IAM and DFT calculations - along the lines marked by the white arrows in (c).

the Lorentz force. Similarly, in a multislice simulation of a ferroelectric crystal, the beam tilt $(\theta)$ due to the polarization of a potential slice can be calculated as the scattering angle due to the Lorentz force acting on the electrons [42]:

$$
\theta=-\frac{e \Delta z P_{R}}{2 U_{0} \varepsilon_{0}}
$$

where $\Delta z$ is the slice thickness, $e$ is the elementary charge, $U_{0}$ is the acceleration voltage, and $P_{R}$ is the remnant polarization. The effect of the mesoscopic field can thus be reproduced by applying such a tiny tilt of the beam during its propagation between subsequent potential slices [Fig. 1(c)]. Since the electric field due to the remnant polarization is typically small, the beam tilt induced by $P_{R}$ is very small compared to typical scattering angles. As an example, in the case of $\mathrm{BiFeO}_{3}$, a large remnant polarization of about $100 \mu \mathrm{C} \mathrm{cm}^{-2}$ corresponds to an electric field of $11.3 \mathrm{~V} \AA^{-1}$. If we set the slice thickness as $\Delta z=3.968 \AA$ (corresponding to one pseudocubic unit cell) the scattering angle due to the mesoscopic ferroelectric polarization of one slice is equal to $\theta=0.061 \mathrm{mrad}$.

The most striking advantage of this second approach is that it allows including the effects of the polar field in the specimen plane overcoming both the periodicity problem that arises when using a modified electrostatic potential and finite sampling in reciprocal space. We thus developed a modified multislice algorithm that allows simulating atomic resolution DPC-STEM signals including the effect of the polarization in the propagation [Fig. 1(c)]. When a uniform mesoscopic electric field exists within the specimen, we can summarize the workflow of the algorithm as (1) calculate the projected potential slices $V_{i}$ (as obtained from IAM or DFT); (2) calculate the interaction of the electron probe with one potential slice $V_{i}$; (3) calculate the beam tilt corresponding to the scattering angle of the electrons under the Lorentz force due to mesoscopic polarization of the crystal slice; (4) calculate the beam propagation between $i$ th and $(i+1)$ th slices, including the polarization beam tilt in the Fresnel propagator (in contrast to the simulation of a tilted propagator describing the crystal tilt, the tilt due to the polarization increases with the number of slices); and (5) iterate through the specimen thickness.

\section{Potential slices (IAM)}

For the independent atom model (IAM), the potential slices used in the multislice simulations were generated starting from the three-dimensional crystal structure, firstly calculating a projection of the structure and then by obtaining the corresponding projected potential. In particular, the crystal structure was created starting from the polar $R 3 c$ phase [43] of $\mathrm{BiFeO}_{3}$. By performing a proper rotation and cut of the structure, it is possible to generate a crystal slab whose dimensions are $4 \times 4 \times 2$ pseudocubic unit cells [Fig. 2(a)]. The choice of the two unit-cell thickness is justified by the fact that it corresponds to the minimum thickness that allows preserving the tilting pattern of the oxygen octahedrons along the $[001]_{P}$ direction. Afterward, we sliced the crystal along the direction parallel to the electron beam propagation-i.e., $[001]_{P}-$ generating two slices (SL1 and SL2) with thickness $\Delta z=$ $3.968 \AA$ [Fig. 2(b)]. Finally, we projected the crystallographic structure of each slice along the $[001]_{P}$ direction, generating two-dimensional projected crystallographic structures.

After generating the projected structures, the projected potentials for the independent atom model were obtained from the elastic scattering factors by Weickenmeier and Kohl within the first-order Born approximation [44]. As an example, Fig. 2(c) shows the IAM total projected potential $\left(V_{\mathrm{SL} 1}+V_{\mathrm{SL} 2}\right)$ along the $[001]_{P}$ direction of $\mathrm{BiFeO}_{3}$.

\section{Potential slices (DFT)}

Using QUANTUM ESPRESSO (QE) code [45,46], we simulated a G-type antiferromagnetic $R 3 c$ BFO cell [47]. PAW pseudopotentials [48] described the core electrons, while a plane-wave basis set the valence. The exchange-correlation was approximated by the PBESol functional [49], and a Hubbard correction of $2 \mathrm{eV}$ was applied to $\mathrm{Fe}$ atoms to catch the proper localization of the $f$ orbitals [50]. Note that 
the electron localization function of the simulated system clearly shows the electron lone pair at the Bi sites (Fig. S1), confirming the accuracy of the obtained electronic solution.

To calculate the total electrostatic potential, we employed the method of Ewald summation [51], as explained in the following. The potential due to nuclear charge was calculated in real space. The all-electron charge was calculated with $\mathrm{QE}$ and its electrostatic potential was calculated together with the screening Gaussians charges of Ewald summation in reciprocal space by fast Fourier transforms. The total electrostatic 3D potential was finally rotated to the correct $[001]_{P}$ orientation via trilinear interpolation and projected onto slices each containing a single atomic layer. For full details on the electronic structure and total potential calculations, we refer the reader to Sec. S1 of Ref. [52].

\section{E. Multislice simulations}

The simulations of the STEM signals (HAADF- and DPCSTEM) were performed using a custom-developed FORTRAN program [53], specifically adapted for the simulations of DPC-STEM. In particular, the simulations were performed setting the parameters to values that resemble the ones commonly used in the experiments, i.e. accelerating voltage: 300 $\mathrm{kV}$; convergence semiangle: $18 \mathrm{mrad}$; electron energy spread: $0.8 \mathrm{eV}$; finite probe size: $0.6 \AA$; real-space sampling: 0.06 and $0.15 \AA$ /pixel; reciprocal space sampling: 0.513 and 1.282 $\mathrm{mrad} /$ pixel; HAADF angular range: $70-160 \mathrm{mrad}$; and DF4 angular range: $10-40 \mathrm{mrad}$.

The segmented-ADF detector was implemented as a four-quadrant detector in agreement with the geometry of a commercially available segmented detector. The thermal vibrations at room temperature are considered in the multislice simulations by averaging 12 frozen phonon configurations [54,55], using the following mean square displacements [56]: $\left\langle u^{2}\right\rangle_{\mathrm{Bi}}=0.064 \AA^{2},\left\langle u^{2}\right\rangle_{\mathrm{Fe}}=$ $0.049 \AA^{2}$, and $\left\langle u^{2}\right\rangle_{\mathrm{O}}=0.081 \AA^{2}$.

Among these parameters, particularly important is the real space sampling, as it is strictly related to the reciprocal space resolution. As the variations we aim to detect in the transmitted disk due to the ferroelectric polarization are rather small, a smaller sampling in the reciprocal space allows having a higher sensitivity in detecting such changes in the reciprocal space. The real space samplings of 0.06 and $0.15 \AA$ Apixel used in this work respectively correspond to 1.282 and 0.513 $\mathrm{mrad} / \mathrm{pixel}$, being the latter value the best choice to detect displacements in the center of mass (CoM) of the transmitted disk as small as fractions of mrad.

The CoM indeed represents a piece of very useful information as it can be directly related to the average momentum transferred to the electron probe by its interaction with the specimen [27]. Interestingly, such a relationship holds also in the case of multiple scattering occurring, e.g., in thick specimens [57]. Therefore, although the momentum transfer can be directly related to the projected electric fields solely for extremely thin specimens [21], it represents a general and powerful means for detecting the effects of polarization and crystal tilt on the differential phase-contrast signal in atomic resolution STEM measurements. In our simulated thickness/defocus series, the fitting of the center of mass of the bright-field disk was performed by calculating its centroid (first image moment), given by

$$
\left\{\operatorname{CoM}_{x}, \operatorname{CoM}_{y}\right\}=\left\{\frac{M_{10}}{M_{00}}, \frac{M_{01}}{M_{00}}\right\}
$$

being the image moments $M_{i j}$ of an $(N \times P)$ image defined by [58]

$$
M_{i j}=\sum_{x}^{N} \sum_{y}^{P} x^{i} y^{j} \times I(x, y) .
$$

\section{F. Specimen tilt}

The specimen misorientation-i.e., the parasitic angular deviation from the nominal on-axis orientation - can play an important role in the DPC-STEM contrast as it can induce strong variations in the intensity distribution within the brightfield disk. We thus took into account the specimen tilt in our IAM multislice simulations and compared its effects to the one of mesoscopic polarization.

As pointed out by Zhou et al. [59], there are in general three approaches to implement the crystal tilt in a multislice simulation that are: (i) tilting the whole supercell, (ii) tilt the electron beam, and (iii) tilt via propagator. Despite the first method is the most demanding from the computational point of view, it is the preferable option as it is the one more closely resembling the experimental situation and because it is more robust for larger angles of tilt.

We thus applied the crystal tilt by creating several supercells of the desired thicknesses and tilting the whole superstructures by the chosen amount of tilt. The superstructures used for the thickness-series simulations were generated by (i) replicating the crystal structure along the $[001]_{P}$ direction to reach the chosen thickness values and (ii) applying a rotation of the structure around the $[1 \overline{1} 0]_{P}$ direction (Fig. S2, Ref. [52]). For each specimen thickness, the projected potential slices were afterward obtained slicing the tilted crystal along the beam propagation direction. In this work, we show thickness-defocus series with crystal tilt values in the range $(0.6,3.6) \mathrm{mrad}$, with intermediate steps every $0.6 \mathrm{mrad}$.

\section{RESULTS AND DISCUSSION}

\section{A. Comparison between the potentials generated by DFT and IAM}

As previously described the potential slices for the multislice simulations were generated using two different approaches, i.e., the independent atom model (IAM) based on the elastic scattering factors by Weickenmeier and Kohl [44], and the density functional theory (DFT). In the IAM case, the potentials of the atoms are calculated as the potential of the atomic species as they are isolated and just juxtaposed within the crystal structure. This approach obviously neglects the charge redistribution effects due to quantum phenomena, like, e.g., localized charges and bonding effects. On the contrary, the DFT approach-despite more demanding from the computational point of view-allows for calculating the electronic structure (ground state) of the many-body system including quantum-mechanical effects. A more accurate description of the electronic state of the system allows building a more precise potential of the crystallographic structure. 

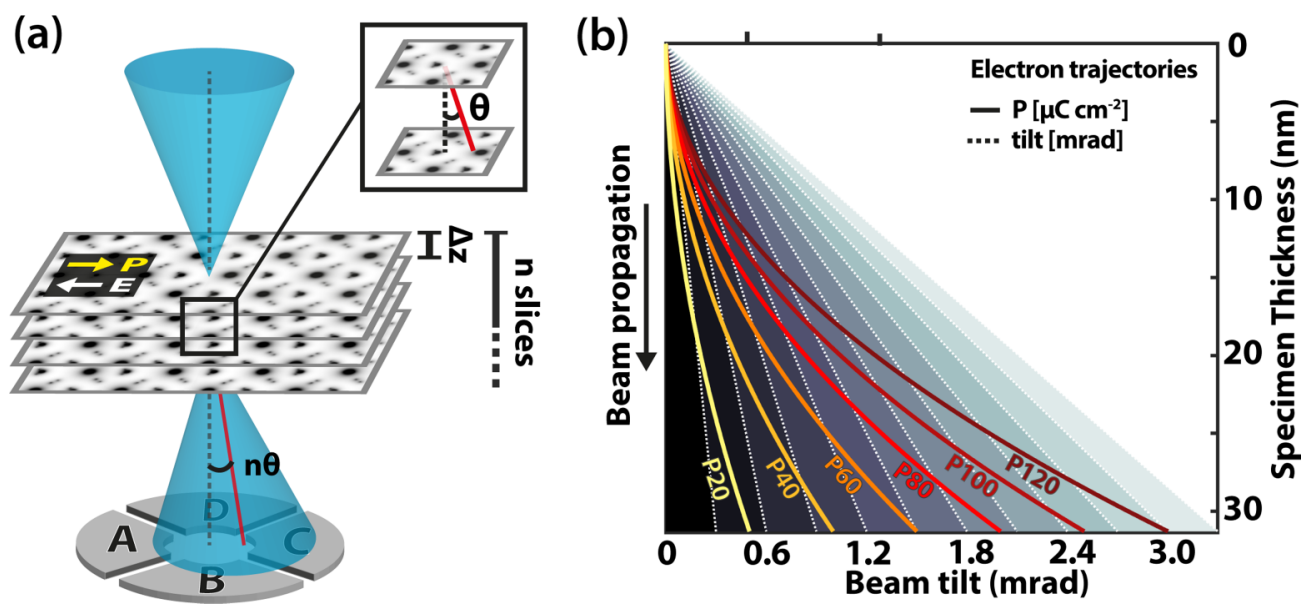

FIG. 3. (a) Schematic representation of the simulated DPC-STEM measurement on a ferroelectric crystal. A convergent electron probe is scanned over the specimen. The ferroelectric polarization induces a beam tilt-detectable using a segmented annular detector. A dynamical treatment of the beam propagation is considered. (b) Evolution of an electron trajectory as it propagates through a ferroelectric crystal (neglecting the channeling effects and multiple scattering). The polarization-induced beam tilt (colored lines) is shown for different polarization values and specimen thicknesses. The white dashed lines serve as a guide for the eye as they depict the trajectories at constant angles spaced $0.3 \mathrm{mrad}$ between each other.

For both the IAM and DFT approaches, we built the potential slices projecting the electrostatic potential along the $[001]_{P}$ direction taking a slice thickness of $3.968 \AA(1$ pseudocubic unit cell). This choice allows a direct comparison of the two potentials. We can compare the IAM and DFT potentials by taking the line profiles along the two directions given by the white arrows in Fig. 2(c). The profiles, shown in Fig. 2(d), highlight very similar trends of the IAM and DFT potentials, with a perfect match of the peaks corresponding to the atomic columns nuclei. Very small differences are visible in the region in between the atomic columns, due to a more precise description of the electronic structure in the DFT calculation.

No remarkable differences are visible for the simulations performed using the potential as obtained under the IAM and DFT calculations (not here reported), in agreement with the prevailing contribution of the atomic nucleus on the atomic potential that governs elastic electron scattering, particularly for medium and heavy elements. While the charge distribution within the atomic nucleus is very localized (length scale $r \approx 10^{-15} \mathrm{~m}$ ), the charge distribution of the electron clouds - responsible for the quantum effects and bondingoccurs over a length scale which is 5 orders of magnitude larger $\left(r \approx 10^{-10} \mathrm{~m}\right)$. These effects might induce very small changes in the potential, which for our purpose are negligible because they are superimposed on the strong potential of the atomic nucleus. However, it is worth noting that such small effects due to the redistribution of electron charge density may become more relevant for light atoms and ultra-thin samples, where the contribution of the nuclei to the electrostatic potential is reduced [60].

\section{B. Thickness and defocus series: Effects of polarization and crystal tilt on DPC contrast}

Figure 3(a) shows a schematic representation of the proposed algorithm for the simulation of high-resolution DPC-
STEM signals of ferroelectric materials. The convergent probe impinges on a specimen, represented in the scheme by the potential slices. In agreement with the described approach, an incremental beam tilt is applied to the propagator after the interaction with each potential slice.

Therefore the electrons propagate through the specimen with a cumulative beam tilt that increases with the specimen thickness. Figure 3(b) shows the evolution of the deflection of an electron trajectory as a function of the specimen thickness for different values of the remnant polarization, assuming the electron is only subject to the mean-field due to the ferroelectric polarization of the crystal (i.e., neglecting channeling effects and dynamical scattering). As visible from the plot, in the case of specimens with thicknesses from a few to tens of nanometers, the total deflection angle due to the mesoscopic polarization is in the range of $10^{-3} \mathrm{rad}$ and can be detected using a segmented detector or pixelated camera.

It is here worth noting that although our approach recalls the previously described rigid-shift model, in our multislice algorithm the beam tilt is applied in the free-space propagation step at each iteration of the multislice routine and it allows a realistic description of the dynamical scattering occurring during the propagation of the beam through the specimen.

The multislice simulations of the atomic resolution DPCSTEM signals were performed as a function of specimen thickness $(z)$ and defocus $(\Delta f)$, with ranges $z=(1.5,32) \mathrm{nm}$ and $\Delta f=(-238,+16) \AA$. First, the simulated HAADF and DPC-STEM signals generated using the IAM approach are shown for comparison in Figs. 4(a) and 4(b), respectively. The signal shows a clear dependency from both thickness and defocus, in agreement with the results previously reported in the literature for the paraelectric $\mathrm{SrTiO}_{3}$ [26]. In particular, the contrast on the DPC amplitude signal quickly degenerates in the in-focus condition with the increase of the thickness already above 10 unit cells $(\sim 40 \AA)$. On the contrary, in underfocus condition, where the nominal focus of the electron probe is within the crystal, the contrast of the amplitude of the 


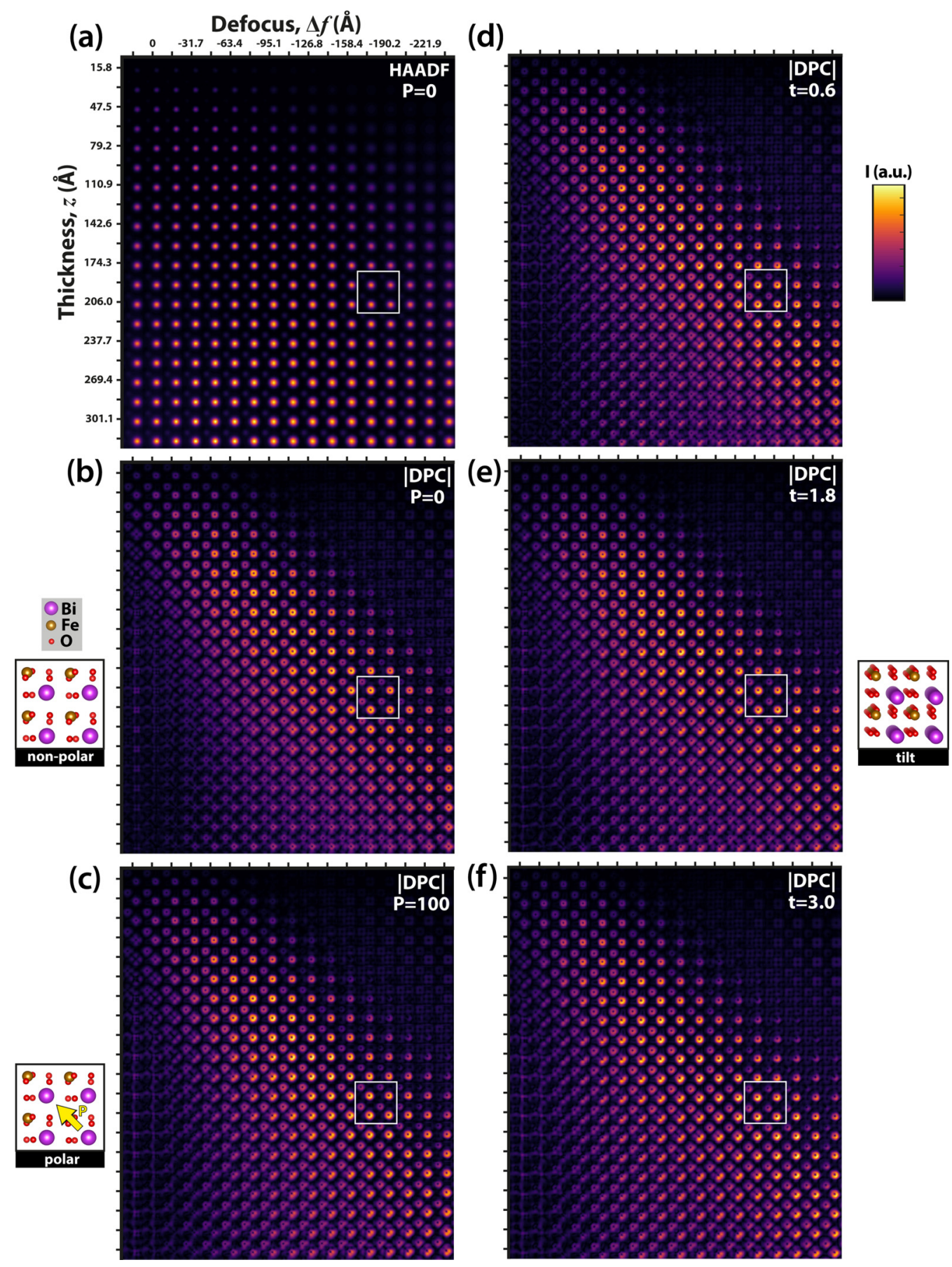

FIG. 4. Thickness and defocus series of the $\mathrm{BiFeO}_{3}$ imaged in the $[001]_{P}$ projection. (a) HAADF signal, (b) DPC amplitude for the nonpolar case $\left(P=0 \mu \mathrm{C} \mathrm{cm}^{-2}\right)$, and (c) DPC amplitude with polar field $\left(P=100 \mu \mathrm{C} \mathrm{cm}^{-2}\right)$. [(d)-(f)] DPC amplitude for the specimen tilt angles of $0.6,1.8$, and $3.0 \mathrm{mrad}$. The thickness and defocus ranges are the same for all the subplots. A different focus and thickness dependence is observed for HAADF and DPC.

atomic electric field preserves its circular shape over a larger thickness up to more than 20 unit cells.

In Fig. 4(c), multislice simulations including the contribution of the mesoscopic polarization $\left(P=100 \mu \mathrm{C} \mathrm{cm}^{-2}\right)$ are shown. A full dataset for different polarization values is given in Fig. S3 of Ref. [52]. Noteworthy, the polar field produces a remarkable change in the DPC amplitude contrast, visible in the image as an asymmetric electric field for the heavy atomic columns (Bi and $\mathrm{Fe}$ ). Although a strong alteration in the shape of the atomic electric field, there is no significant variation in the signal dependency on specimen thickness and defocus with respect to the nonpolar case. The asymmetries observed in the DPC amplitude indicate that the effect of the polarization field considerably alters the beam channeling conditions and, consequently, the contrast of the atomic-resolution DPC signal. The technique is thus sensitive to polar fields. 
An effect similar to the one induced by the specimen polarization can arise as a consequence of specimen misorientation, that might occur in the case the zone axis of the specimen is not properly oriented along the direction of the optical axis of the microscope. To investigate the effect of crystal tilt, we simulated defocus and thickness series applying a tilt of the specimen in the range 0.6 to $3.6 \mathrm{mrad}$. The effect of specimen tilt is shown in Figs. 4(d)-4(f) for three different tilt angles of $0.6,1.8$, and $3.0 \mathrm{mrad}$ (for the full dataset see Fig. S4 of Ref. [52]).

Although the effects of polarization and specimen tilt are qualitatively similar, we can perform an accurate comparison by plotting the line profiles of the DPC amplitude for the different cases. For the comparison, we selected the simulated signals obtained setting the thickness $z=20.6 \mathrm{~nm}$ and defocus $\Delta f=-174 \AA$ (corresponding to the area marked by the white box in Fig. 4). For instance, Figs. 5(a)-5(c) show the magnified view of the simulated signals for three different cases, i.e., (i) $P=0$, (ii) $P=100 \mu \mathrm{C} \mathrm{cm}^{-2}$, and (iii) crystal tilt $t=1.8 \mathrm{mrad}$, respectively.

The asymmetric electric fields for the heavy atomic columns are detected solely in the signals of the polar and tilted specimens. Interestingly, at first sight, no fingerprint of the unit cell dipole is visible in the DPC-STEM signal for the nonpolar case. The contribution due to the atomic displacements is indeed very small and its effects can be disclosed by a proper choice of the image equalization (see Fig. S5 in Ref. [52] for more details).

By taking the line profiles for all the signals along the white dashed area marked in Fig. 5(a), we can directly compare such effects. Interestingly, the line profiles for different polarization values [shown in Fig. 5(d)] demonstrate that in the polar case the asymmetry of the Bi columns increases more rapidly compared to the $\mathrm{Fe}$ ones. On the contrary, in the case of specimen tilt the profiles of $\mathrm{Fe}$ and $\mathrm{Bi}$ atomic columns [Fig. 5(e)] show similar trends. The features induced by the ferroelectric polarization on the atomic electric fields are in very good agreement with the ones observed in previously reported experimental data, where asymmetries of the atomic electric field were observed in the polar regions of a thin film alternating with nonpolar domains [31].

We can further study the differences between the effects of polarization and specimen tilt by analyzing the corresponding simulated CBED patterns. Figure 5(f) shows the simulated diffraction pattern for the nonpolar case. The diffraction pattern was obtained by averaging the CBED pattern over the different scan positions on an area covering one pseudocubic unit cell of $\mathrm{BiFeO}_{3}$. The plots in Figs. $5(\mathrm{~g})$ and 5(h) show the difference between the averaged CBED patterns for the polar $\left(P=100 \mu \mathrm{C} \mathrm{cm}^{-2}\right)$ and tilted $(t=1.8$ mrad) cases and their references (i.e., the nonpolar $P=0$ and untilted $t=0$, respectively). A clear variation of the center of mass of the transmitted disk occurs in both cases. The change in the diffraction pattern looks overall similar, but the subtle variations in the line profiles given Figs. 5(i) and $5(\mathrm{j})$ [the line profiles are taken along the black arrows in Figs. 5(g) and 5(h)] suggest that it is possible to discriminate between the mesoscopic polarization and specimen tilt by analyzing the intensity distribution in the CBED pattern.
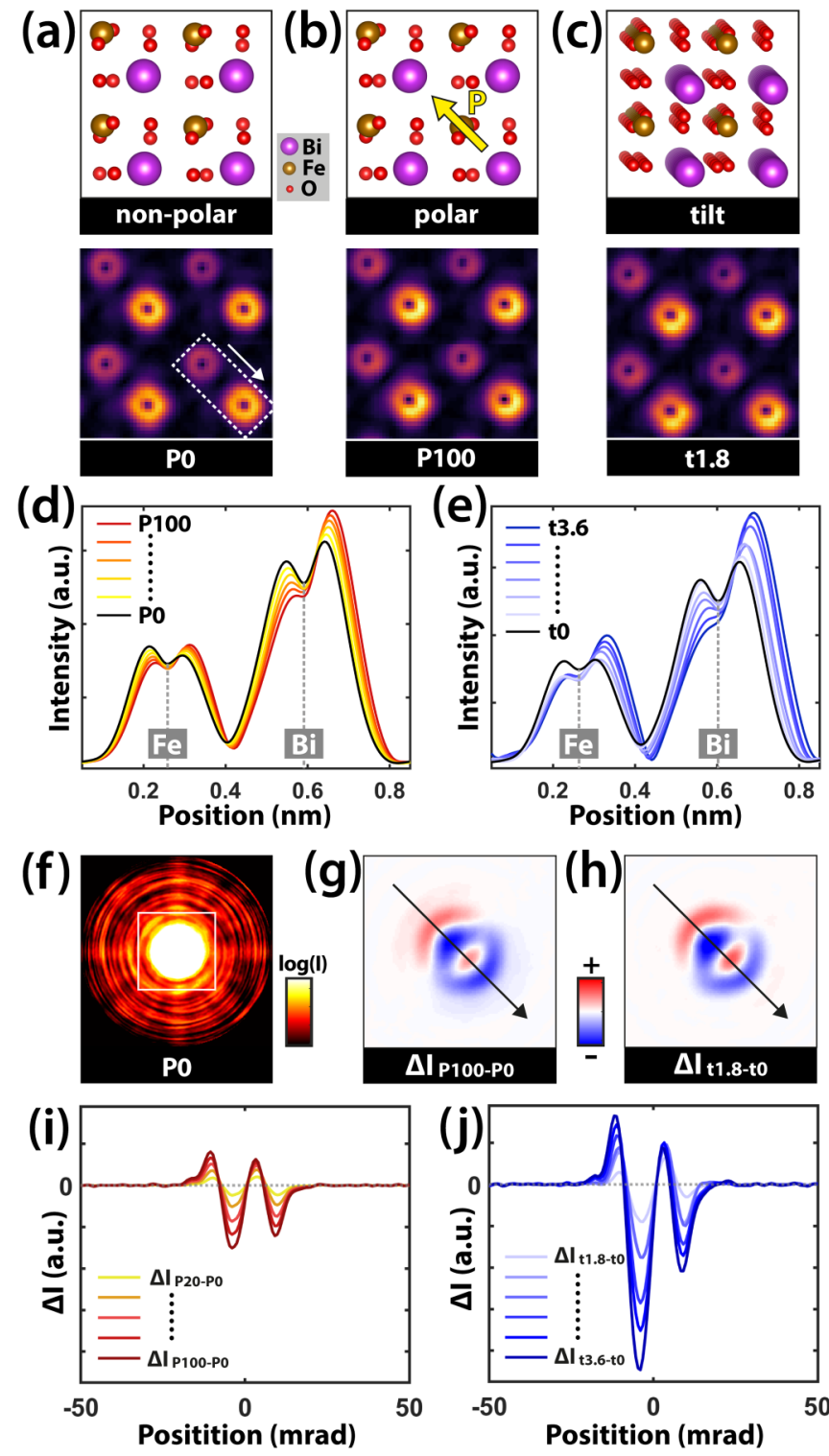

FIG. 5. Sketches of the crystal structures in the $[001]_{P}$ zone axis for the three cases of (a) nonpolar, (b) polar and (c) tilted specimens. The yellow arrow gives the direction of the polarization. The magnified views of the simulated DPC amplitude for the cases $P=0, P=$ $100 \mu \mathrm{C} \mathrm{cm}^{-2}$, and $t=1.8 \mathrm{mrad}$ (corresponding to the areas marked by the white boxes in Fig. 4 , i.e., $z=20.6 \mathrm{~nm}$ and $\Delta f=-174 \AA$ ) are shown below the sketches. (d) Line profiles obtained integrating the DPC amplitude with mesoscopic polarization over an area like the one marked by the white dashed box shown in (a). (e) Similar line profiles obtained integrating the DPC amplitude for different values of crystal tilt angle. (f) Simulated CBED pattern for the nonpolar specimen $(z=20.6 \mathrm{~nm}$ and $\Delta f=-174 \AA)$. Difference of CBED patterns of the $(\mathrm{g})$ polar $\left(P=100 \mu \mathrm{C} \mathrm{cm}^{-2}\right)$ and $(\mathrm{h})$ tilted $(t=$ $1.8 \mathrm{mrad}$ ) specimens with respect to the reference one. Comparison of line profiles for the different patterns for the (i) polar and (j) tilted cases, taken along the black arrows.

\section{Discrimination between mesoscopic polar field and specimen tilt}

Due to the similarity between the effects induced by mesoscopic polarization and crystal tilt, a challenging aspect of the 


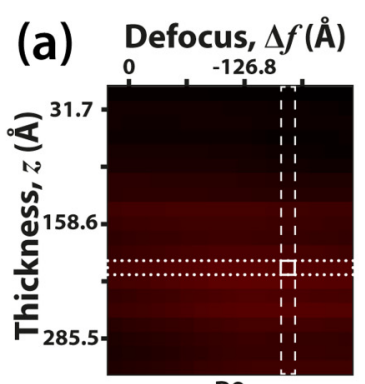

PO

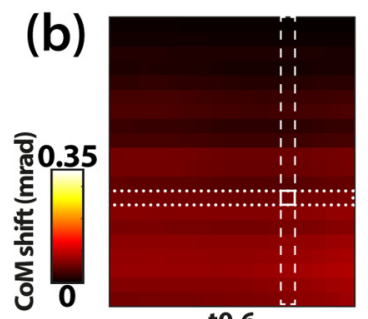

t0.6

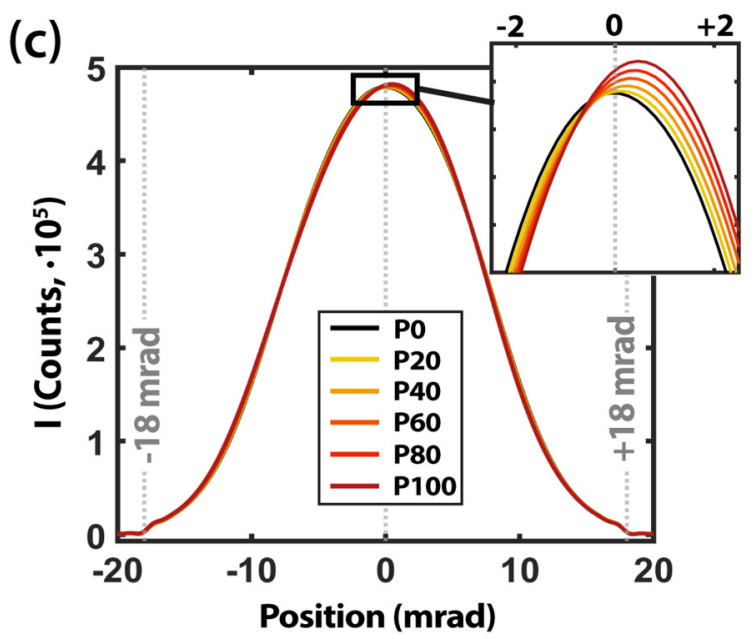

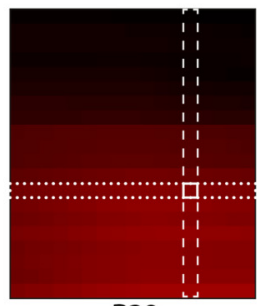

P20

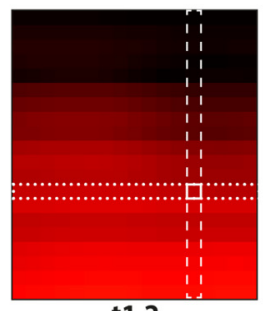

t1.2

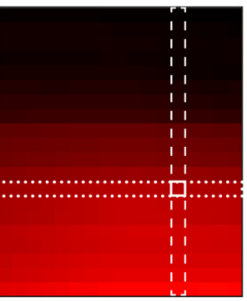

P40

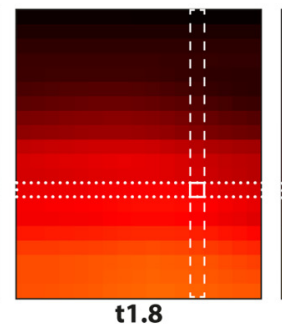

$+2$

\begin{abstract}
$+2$
\end{abstract}
(d)
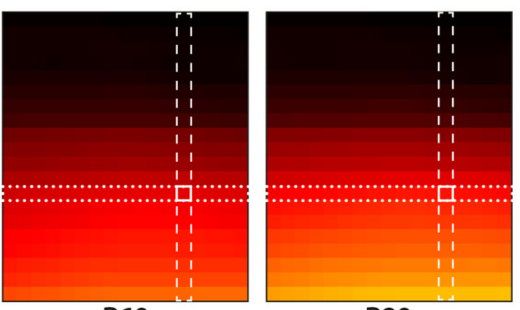

P80

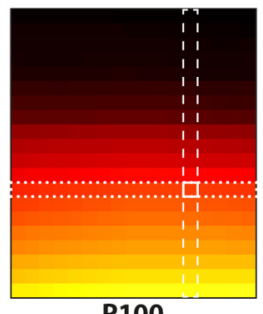

P60
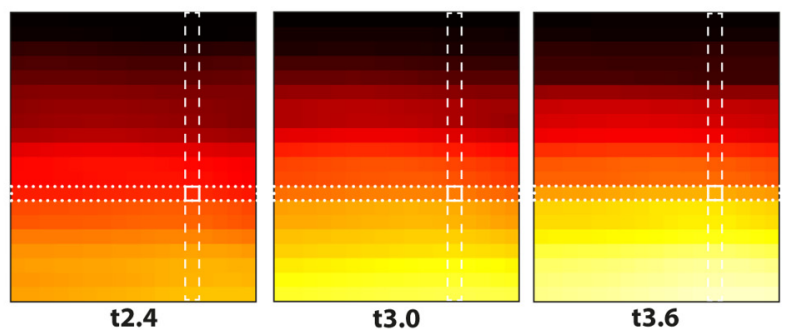

t3.6

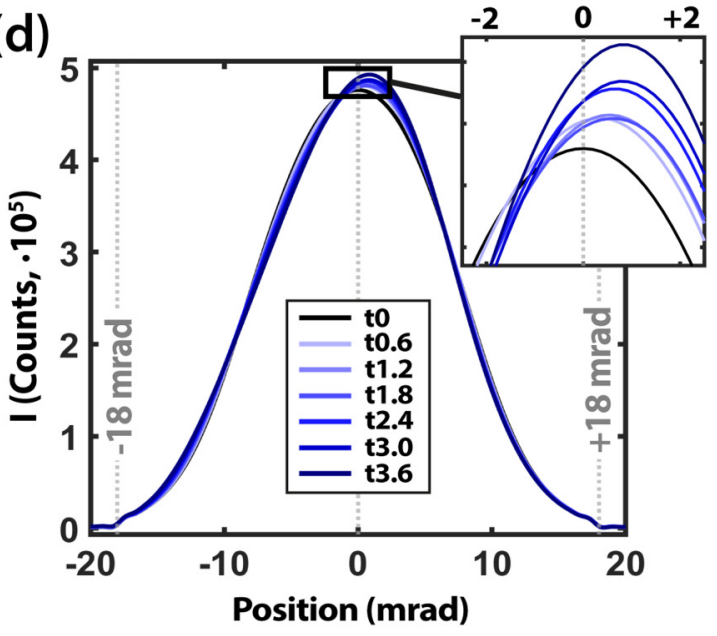

FIG. 6. Thickness and defocus dependent maps of the average shift of the CoM for (a) different values of mesoscopic polarization and (b) crystal tilt angles. [(c) and (d)] Line profiles of the bright field disks obtained for a thickness $z=20.6 \mathrm{~nm}$ and a defocus $\Delta f=-174.3 \AA$, with mesoscopic polarization and crystal tilt, respectively. The analyzed bright field disk corresponds to the value marked by the white box in (a) and (b). The magnified views on the right show the relative positions of the peaks for the different polarization and tilt values.

DPC-STEM technique is the discrimination between the two of them. A possible approach relies on analyzing the brightfield disk by fitting its center of mass (CoM). Figure 6(a) shows the maps of the CoM shift for the different polarization values. Each pixel of the maps represents the CoM shift of the bright field disk obtained as average over the raster scan positions covering one unit cell. The maps are calculated for the same ranges of thickness and defocus as the simulated DPC-STEM data in Fig. 4. Similar maps are obtained for the specimen tilts and reported in Fig. 6(b).

By comparing the CoM shifts, we observe different trends with respect to defocus and thickness. The reduced values of the CoM shifts (by an order of 10 compared to the calculated rigid shifts) confirm that the dynamical electron beam propagation generally induces complex intensity redistribution in the CBED pattern and not a simple rigid shift of the brightfield disk.

To better understand the different trends in the CoM shifts, we can analyze the bright-field disk by taking a line profile along the direction of polarization and specimen tilt. Figures 6(c) and 6(d) show the line profiles of the bright-field disk for the specimen thickness $z=20.6 \mathrm{~nm}$ and defocus $\Delta f=-174 \AA$ [corresponding to the area marked by the white square box in Figs. 6(a) and 6(b)]. Despite the similar shape of the curves, the magnified views of the central parts of their profiles highlight the different effects of polarization and tilt on the shift of the CoM.

This diverse trend, previously presented in Figs. 5(g)-5(j) as a differential plot, further confirms that we can make use of the different CoM dependence on specimen thickness and defocus to discriminate between the specimen polarization and crystal tilt. In particular, we can distinguish between the polarization and specimen tilt by acquiring a series of CBED patterns at different defoci and fitting the CoM of their bright-field disks. In practice, recording such focal series correspond to analyze the trend of the CoM shift along a row (at a given thickness) in Figs. 6(a) and 6(b). As an example, if we consider the CoM shifts for a specimen thickness of 

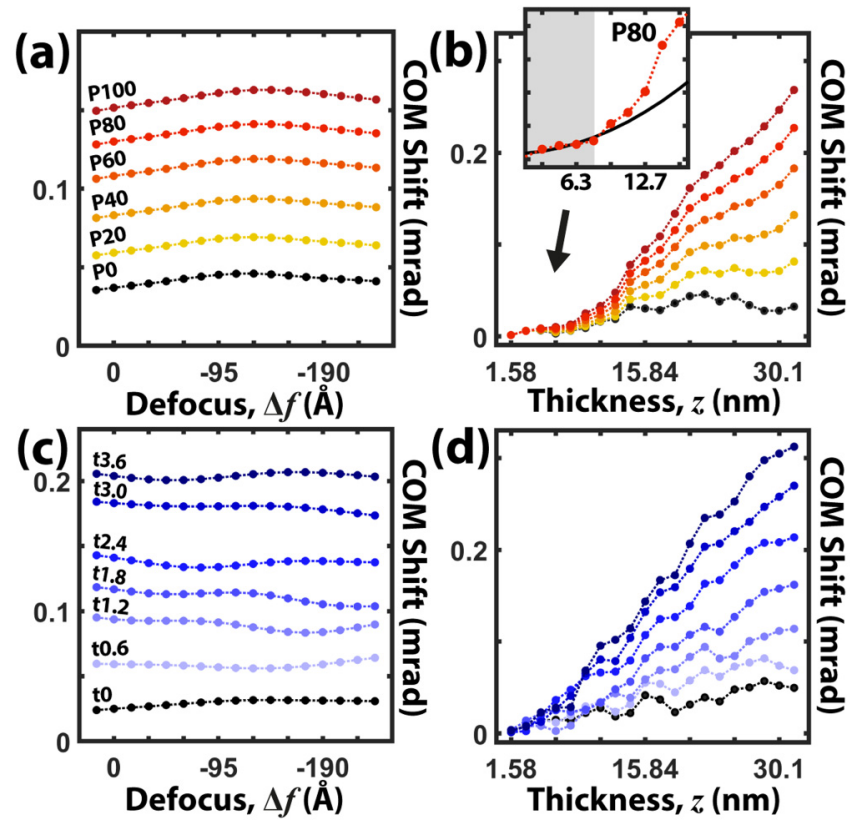

FIG. 7. Line profiles of the CoM shifts for the different polarization and tilt values. (a) Defocus-dependent line profiles of the CoM shifts for different polarization values. The line profiles are taken at the constant specimen thickness $z=20.6 \mathrm{~nm}$ [dotted rectangles in Fig. 5(a)]. (b) Thickness-dependent line profiles of the CoM shifts for the different polarization values, taken at constant defocus $\Delta f=$ $-174.3 \AA$ [dashed rectangles in Fig. 5(a)]. The inset in (b) shows the comparison of the curves of $P=80$ (red) and the rigid-shift model (black). [(c) and (d)] Similarly obtained line profiles for the various specimen tilt angles.

$z=20.6 \mathrm{~nm}$ [dashed rectangular box in Figs. 6(a) and 6(b)], we can directly compare the defocus dependence of the CoM shifts for the different cases. The line profiles are shown in Figs. 7(a) and 7(c) for the mesoscopic polarization and specimen tilt. In the polar case, the CoM shifts show a maximum at $\Delta f \approx-120 \AA$ for all the different polarization values. This trend is not observed in the specimen tilt case and thus we can exploit the defocus-induced variation of bright-field CoM to detect the polar properties of the specimen.

We can now perform a similar analysis by taking the line profiles at constant defocus $\Delta f=-174.3 \AA$ and studying the thickness-dependent variation of the CoM in the two cases. The line profiles in Figs. 7(b) and 7(d) highlight significant differences especially at the lower thicknesses, where the polar case shows a nonlinear trend. In particular, if we observe, e.g., the low-thickness trend for the curve corresponding to $P=80 \mu \mathrm{C} \mathrm{cm}^{-2}$ [see inset in Fig. 7(b)], we can observe that it perfectly matches the curve calculated using the rigid-shift model for specimen thickness up to $\sim 7 \mathrm{~nm}$ (about 15 unit cells). A strong deviation-due to the dynamical diffractionis instead observed for higher thickness values, marking the failure of the rigid-shift model in the description of the electron beam propagation through a polar ferroelectric specimen.

\section{CONCLUSIONS}

Our work explores the origin of the contrast of highresolution DPC-STEM in ferroelectric materials, where a net remnant polarization exists. Previous experimental results showed that high-resolution DPC-STEM data are sensitive to polar fields in ferroelectric specimens, highlighting the important role that DPC-STEM can play in the atomic-scale characterization of such materials. The proper interpretation of the experimental high-resolution DPC-STEM, however, requires a detailed comparison with image simulations. Indeed, the effects of dynamical diffraction (like e.g. multiple scattering and beam channeling) complicate the analysis of the DPC-STEM data, as the rigid-shift model fails to describe the real variation in the CBED pattern. Since the mesoscopic polar field induces a tilt to the electron wave function, the eventual residual specimen tilt could also affect the contrast of the DPC-STEM signal.

Here we propose a modified multislice algorithm that allows taking into account the mesoscopic polarization by a mean-field approach, i.e., including the average field generated by the polarization of the whole crystal as an additive term. Since the multislice algorithm makes extensive use of Fourier transforms, a convenient approach to implement this modified algorithm is to include the contribution of remnant polarization in the free-space Fresnel propagator of the multislice flowchart as a small, incrementally increasing tilt of the electron wave function.

The proposed method allows for simulating highresolution DPC-STEM data for different values of polarization, specimen thickness, and specimen tilt. The results, which are in agreement with experimental data [26], demonstrate that the DPC-STEM signal is strongly affected by the polar state of the specimen and it shows a complex dependence on the specimen thickness and the defocus.

Specifically, the mesoscopic polar field leads to a marked asymmetry in the detected atomic electric field that reconciles with the previously reported experimental results [31]. Our results suggest that, for specimen thicknesses in the range of 10-30 nm, high-resolution DPC-STEM is sensitive to polar fields of $40 \mu \mathrm{C} \mathrm{cm}^{-2}$.

A similar effect, yet different in its intensity, can be generated by specimen tilt. Such difference, originating from the different channeling conditions-i.e., an increasing cumulative tilt for the electron wave function in the case of mesoscopic polarization, oppositely to the constant angle in the case of crystal tilt-is responsible for a different dependence of the CBED pattern on the two different effects. In particular, the different dependence of the CoM shift on the defocus for the case of mesoscopic polarization and specimen tilt allows for discriminating between the two effects by acquiring a focal series of CBED patterns, possible using a 2D camera or a pixel-array detector.

Moreover, the thickness-dependent variation of the CoM shift demonstrates that for thin polar specimens-e.g., specimens whose thickness is lower than $7 \mathrm{~nm}$ for the $P=$ $80 \mu \mathrm{C} \mathrm{cm}^{-2}$ case - the rigid-shift model is effective in describing the effect of mesoscopic polarization on the DPCSTEM signal. On the contrary, when the specimen's thickness exceeds this value, the dynamical scattering contribution becomes dominant, i.e., although the asymmetries in the detected atomic electric fields persist, the rigid-shift model fails in quantitatively describing the observed contrast of DPC-STEM data. In the latter case, the quantification of the 
experimental data, therefore, strictly requires a treatment that includes dynamical diffraction.

This work represents a proof-of-concept of the suitability of atomic-resolution differential phase-contrast techniques for the investigation of complex polar materials and, at the same time, defines its applicability limits. These results represent a significant step forward in the study of ferroelectric materials using differential phase-contrast STEM, further breeding the ground for 4D-STEM methods.

\section{ACKNOWLEDGMENTS}

The following institutions financially supported this work: Swiss National Science Foundation under Project No. 200021_175926 (M.C. and M.D.R.), the EU European Research Council ERC Grant No. 681312 (R.E. and M.B.), and the InnoSuisse Innovation Project ENG No. 35839.1 (M.C. and R.E.). The authors are grateful to Daniele Passerone for fruitful discussions about the computational aspects of firstprinciple calculations.
[1] P. D. Nellist, M. F. Chisholm, N. Dellby, O. L. Krivanek, M. F. Murfitt, Z. S. Szilagyi, A. R. Lupini, A. Borisevich, W. H. Sides, and S. J. Pennycook, Science 305, 1741 (2004).

[2] R. Erni, M. D. Rossell, C. Kisielowski, and U. Dahmen, Phys. Rev. Lett. 102, 096101 (2009).

[3] S. J. Pennycook, and P. D. Nellist, Scanning Transmission Electron Microscopy - Imaging and Analysis (Springer, New York, 2011), pp. 91-115.

[4] N. Shibata, S. D. Findlay, Y. Kohno, H. Sawada, Y. Kondo, and Y. Ikuhara, Nat. Phys. 8, 611 (2012).

[5] H. Yang, L. Jones, H. Ryll, M. Simson, H. Soltau, Y. Kondo, R. Sagawa, H. Banba, I. MacLaren, and P. D. Nellist, J. Phys. Conf. Ser. 644, 012032 (2015).

[6] H. Rose, Optik 39, 416 (1974).

[7] N. H. Dekkers and H. De Lang, Optik 41, 452 (1974).

[8] M. Haider, A. Epstein, P. Jarron, and C. Boulin, Ultramicroscopy 54, 41 (1994).

[9] M. Hammel and H. Rose, Ultramicroscopy 58, 403 (1995).

[10] C. Ophus, P. Ercius, M. Huijben, and J. Ciston, Appl. Phys. Lett. 110, 063102 (2017).

[11] E. Yücelen, I. Lazić, and E. G. T. Bosch, Sci. Rep. 8, 2676 (2018).

[12] A. K. Shukla, C. Ophus, C. Gammer, and Q. Ramasse, Microsc. Microanal. 22, 494 (2016).

[13] O.-H. Kwon, B. Barwick, H. S. Park, J. S. Baskin, and A. H. Zewail, Nano Lett. 8, 3557 (2008).

[14] B. Barwick, H. S. Park, O.-H. Kwon, J. S. Baskin, and A. H. Zewail, Science 322, 1227 (2008).

[15] P. Cueva, E. Padget, and D. A. Muller, Microsc. Microanal. 25, 1934 (2019).

[16] A. C. Meng, M. R. Braun, Y. Wang, C. S. Fenrich, M. Xue, D. R. Diercks, B. P. Gorman, M.-I. Richard, A. F. Marshall, W. Cai, J. S. Harris, and P. C. McIntyre, Mater. Today Nano 5, 100026 (2019)

[17] N. Shibata, T. Seki, G. Sánchez-Santolino, S. D. Findlay, Y. Kohno, T. Matsumoto, R. Ishikawa, and Y. Ikuhara, Nat. Commun. 8, 15631 (2017).

[18] N. Shibata, S. D. Findlay, H. Sasaki, T. Matsumoto, H. Sawada, Y. Kohno, S. Otomo, R. Minato, and Y. Ikuhara, Sci. Rep. 5, 10040 (2015).

[19] T. Matsumoto, Y. G. So, Y. Kohno, Y. Ikuhara, and N. Shibata, Nano Lett. 18, 754 (2018).

[20] T. Matsumoto, Y.-G. So, Y. Kohno, H. Sawada, Y. Ikuhara, and N. Shibata, Sci. Adv. 2, e1501280 (2016).

[21] T. Seki, G. Sánchez-Santolino, R. Ishikawa, S. D. Findlay, Y. Ikuhara, and N. Shibata, Ultramicroscopy 182, 258 (2017).
[22] H. G. Brown, N. Shibata, H. Sasaki, T. C. Petersen, D. M. Paganin, M. J. Morgan, and S. D. Findlay, Ultramicroscopy 182, 169 (2017).

[23] J. A. Hachtel, J. C. Idrobo, and M. Chi, Adv. Struct. Chem. Imaging 4, 10 (2018).

[24] I. MacLaren, L. Q. Wang, D. McGrouther, A. J. Craven, S. McVitie, R. Schierholz, A. Kovács, J. Barthel, and R. E. DuninBorkowski, Ultramicroscopy 154, 57 (2015).

[25] T. Sannomiya, Y. Haga, Y. Nakamura, O. Nittono, and Y. Takahashi, J. Appl. Phys. 95, 214 (2004).

[26] R. Close, Z. Chen, N. Shibata, and S. D. Findlay, Ultramicroscopy 159, 124 (2015).

[27] K. Müller, F. F. Krause, A. Béché, M. Schowalter, V. Galioit, S. Löffler, J. Verbeeck, J. Zweck, P. Schattschneider, and A. Rosenauer, Nat. Commun. 5, 5653 (2014).

[28] M. Campanini, M. Trassin, C. Ederer, R. Erni, and M. D. Rossell, ACS Appl. Electron. Mater. 1, 1019 (2019).

[29] J. Zweck, F. Schwarzhuber, J. Wild, and V. Galioit, Ultramicroscopy 168, 53 (2016).

[30] A. Lubk and J. Zweck, Phys. Rev. A - At. Mol. Opt. Phys. 91, 23805 (2015).

[31] M. Campanini, R. Erni, C.-H. C. H. Yang, R. Ramesh, and M. D. M. D. Rossell, Nano Lett. 18, 717 (2018).

[32] E. J. Kirkland, Advanced Computing in Electron Microscopy, 2nd ed., (Springer, Boston (MA), USA, 2010).

[33] D. Lebeugle, D. Colson, A. Forget, and M. Viret, Appl. Phys. Lett. 91, 022907 (2007).

[34] H. W. Jang, S. H. Baek, D. Ortiz, C. M. Folkman, R. R. Das, Y. H. Chu, P. Shafer, J. X. Zhang, S. Choudhury, V. Vaithyanathan, Y. B. Chen, D. A. Felker, M. D. Biegalski, M. S. Rzchowski, X. Q. Pan, D. G. Schlom, L. Q. Chen, R. Ramesh, and C. B. Eom, Phys. Rev. Lett. 101, 107602 (2008).

[35] K. Y. Yun, M. Noda, and M. Okuyama, Appl. Phys. Lett. 83, 3981 (2003).

[36] J. Dho, X. Qi, H. Kim, J. L. MacManus-Driscoll, and M. G. Blamire, Adv. Mater. 18, 1445 (2006).

[37] F. Yan, T. J. Zhu, M. O. Lai, and L. Lu, Scr. Mater. 63, 780 (2010).

[38] P. Ravindran, R. Vidya, A. Kjekshus, H. Fjellvåg, and $\mathrm{O}$. Eriksson, Phys. Rev. B 74, 224412 (2006).

[39] C. Ederer and N. A. Spaldin, Phys. Rev. B 71, 060401 (2005).

[40] H. Lichte, M. Reibold, K. Brand, and M. Lehmann, Ultramicroscopy 93, 199 (2002).

[41] M. Fiebig, T. Lottermoser, D. Meier, and M. Trassin, Nat. Rev. Mater. 1, 16046 (2016). 
[42] I. Lazić and E. G. T. Bosch, Adv. Imaging Electron Phys. 199, 75 (2017).

[43] J. M. Moreau, C. Michel, R. Gerson, and W. J. James, J. Phys. Chem. Solids 32, 1315 (1971).

[44] A. Weickenmeier and H. Kohl, Acta Crystallogr. Sect. A 47, 590 (1991).

[45] P. Giannozzi, S. Baroni, N. Bonini, M. Calandra, R. Car, C. Cavazzoni, D. Ceresoli, G. L. Chiarotti, M. Cococcioni, I. Dabo, A. Dal Corso, S. De Gironcoli, S. Fabris, G. Fratesi, R. Gebauer, U. Gerstmann, C. Gougoussis, A. Kokalj, M. Lazzeri, L. Martin-Samos, N. Marzari, F. Mauri, R. Mazzarello, S. Paolini, A. Pasquarello, L. Paulatto, C. Sbraccia, S. Scandolo, G. Sclauzero, A. P. Seitsonen, A. Smogunov, P. Umari, and R. M. Wentzcovitch, J. Phys.: Condens. Matter 21, 395502 (2009).

[46] P. Giannozzi, O. Andreussi, T. Brumme, O. Bunau, M. Buongiorno Nardelli, M. Calandra, R. Car, C. Cavazzoni, D. Ceresoli, M. Cococcioni, N. Colonna, I. Carnimeo, A. Dal Corso, S. De Gironcoli, P. Delugas, R. A. Distasio, A. Ferretti, A. Floris, G. Fratesi, G. Fugallo, R. Gebauer, U. Gerstmann, F. Giustino, T. Gorni, J. Jia, M. Kawamura, H. Y. Ko, A. Kokalj, E. Kücükbenli, M. Lazzeri, M. Marsili, N. Marzari, F. Mauri, N. L. Nguyen, H. V. Nguyen, A. Otero-De-La-Roza, L. Paulatto, S. Poncé, D. Rocca, R. Sabatini, B. Santra, M. Schlipf, A. P. Seitsonen, A. Smogunov, I. Timrov, T. Thonhauser, P. Umari, N. Vast, X. Wu, and S. Baroni, J. Phys.: Condens. Matter 29, 465901 (2017).

[47] J. K. Shenton, D. R. Bowler, and W. L. Cheah, J. Phys.: Condens. Matter 29, 445501 (2017).

[48] P. E. Blöchl, Phys. Rev. B 50, 17953 (1994).

[49] J. P. Perdew, A. Ruzsinszky, G. I. Csonka, O. A. Vydrov, G. E. Scuseria, L. A. Constantin, X. Zhou, and K. Burke, Phys. Rev. Lett. 100, 136406 (2008).
[50] R. J. Zeches, M. D. Rossell, J. X. Zhang, A. J. Hatt, Q. He, C.-H. Yang, A. Kumar, C. H. Wang, A. Melville, C. Adamo, G. Sheng, Y.-H. Chu, J. F. Ihlefeld, R. Erni, C. Ederer, V. Gopalan, L. Q. Chen, D. G. Schlom, N. A. Spaldin, L. W. Martin, and R. Ramesh, Science 326, 977 (2009).

[51] D. Frenkel and B. Smit, Understanding Molecular Simulation, Computational Science Series Vol 1 (Academic Press, Orlando (FL), USA, 2001).

[52] See Supplemental Material at http://link.aps.org/supplemental/ 10.1103/PhysRevB.101.184116 for more details about IAM/DFT potential calculation, the full dataset of DPC-STEM simulations, the study of the local dipole on the DPC-STEM data.

[53] R. Erni, H. Heinrich, and G. Kostorz, Ultramicroscopy 94, 125 (2003).

[54] D. A. Muller, B. Edwards, E. J. Kirkland, and J. Silcox, Ultramicroscopy 86, 371 (2001).

[55] R. Erni, Ph.D. thesis, Swiss Federal Institute of Technology Zürich, 2003, available at https://doi.org/10.3929/ethz-a004489839.

[56] A. Palewicz, R. Przeniosło, I. Sosnowska, and A. W. Hewat, Acta Crystallogr. Sect. B Struct. Sci. 63, 537 (2007).

[57] K. Müller-Caspary, F. F. Krause, F. Winkler, A. Béché, J. Verbeeck, S. Van Aert, and A. Rosenauer, Ultramicroscopy 203, 95 (2019).

[58] M. R. Teague, J. Opt. Soc. Am. 70, 920 (1980).

[59] D. Zhou, K. Müller-Caspary, W. Sigle, F. F. Krause, A. Rosenauer, and P. A. van Aken, Ultramicroscopy 160, 110 (2016).

[60] K. Müller-Caspary, M. Duchamp, M. Rösner, V. Migunov, F. Winkler, H. Yang, M. Huth, R. Ritz, M. Simson, S. Ihle, H. Soltau, T. Wehling, R. E. Dunin-Borkowski, S. Van Aert, and A. Rosenauer, Phys. Rev. B 98, 121408(R) (2018). 